\title{
TU/e emonown

\section{Coordination of manufacturing, remanufacturing and returns acceptance in hybrid manufacturing/remanufacturing systems}

\section{Citation for published version (APA):}

Vercraene, S., Gayon, J-P., \& Flapper, S. D. P. (2014). Coordination of manufacturing, remanufacturing and returns acceptance in hybrid manufacturing/remanufacturing systems. International Journal of Production Economics, 148, 62-70. https://doi.org/10.1016/j.ijpe.2013.11.001

DOI:

10.1016/j.ijpe.2013.11.001

Document status and date:

Published: 01/01/2014

\section{Document Version:}

Publisher's PDF, also known as Version of Record (includes final page, issue and volume numbers)

\section{Please check the document version of this publication:}

- A submitted manuscript is the version of the article upon submission and before peer-review. There can be important differences between the submitted version and the official published version of record. People interested in the research are advised to contact the author for the final version of the publication, or visit the $\mathrm{DOI}$ to the publisher's website.

- The final author version and the galley proof are versions of the publication after peer review.

- The final published version features the final layout of the paper including the volume, issue and page numbers.

Link to publication

\section{General rights}

Copyright and moral rights for the publications made accessible in the public portal are retained by the authors and/or other copyright owners and it is a condition of accessing publications that users recognise and abide by the legal requirements associated with these rights.

- Users may download and print one copy of any publication from the public portal for the purpose of private study or research.

- You may not further distribute the material or use it for any profit-making activity or commercial gain

- You may freely distribute the URL identifying the publication in the public portal.

If the publication is distributed under the terms of Article 25fa of the Dutch Copyright Act, indicated by the "Taverne" license above, please follow below link for the End User Agreement:

www.tue.nl/taverne

Take down policy

If you believe that this document breaches copyright please contact us at:

openaccess@tue.nl

providing details and we will investigate your claim. 


\title{
Coordination of manufacturing, remanufacturing and returns acceptance in hybrid manufacturing/remanufacturing systems
}

\author{
Samuel Vercraene ${ }^{a}$, Jean-Philippe Gayon ${ }^{\mathrm{b}, *}$, Simme Douwe Flapper $^{\mathrm{c}}$ \\ a INSA-Lyon, DISP, Villeurbanne F-69621, France \\ ${ }^{\mathrm{b}}$ Grenoble-INP/UJF-Grenoble 1/CNRS, G-SCOP UMR5272 Grenoble, F-38031 France \\ ${ }^{\mathrm{c}}$ Technische Universiteit Eindhoven, P.O. Box 513, 5600 MB Eindhoven, Netherlands
}

\section{A R T I C L E I N F O}

Article history:

Received 21 December 2012

Accepted 4 November 2013

Available online 15 November 2013

Keywords:

Remanufacturing

Manufacturing

Returns acceptance control

Inventory control

Stochastic dynamic programming

Optimal policy

Heuristic policies

\begin{abstract}
A B S T R A C T
This paper deals with the coordination of manufacturing, remanufacturing and returns acceptance control in a hybrid production-inventory system. We use a queuing control framework, where manufacturing and remanufacturing are modelled by single servers with exponentially distributed processing times. Customer demand and returned products arrive in the system according to independent Poisson processes. A returned product can be either accepted or rejected. When accepted, a return is placed in a remanufacturable product inventory. Customer demand can be satisfied as well by new and remanufactured products. The following costs are included: stock keeping, backorder, manufacturing, remanufacturing, acceptance and rejection costs. We show that the optimal policy is characterized by two state-dependent base-stock thresholds for manufacturing and remanufacturing and one state-dependent return acceptance threshold. We also derive monotonicity results for these thresholds. Based on these theoretical results, we introduce several relevant heuristic control rules for manufacturing, remanufacturing and returns acceptance. In an extensive numerical study we compare these policies with the optimal policy and provide several insights.
\end{abstract}

(c) 2013 Elsevier B.V. All rights reserved.

\section{Introduction}

During the last two decades, quite some attention has been paid to the problem of jointly controlling the manufacturing of new products and remanufacturing of returned products. In addition to the joint control of manufacturing and remanufacturing, another important issue is whether or not to accept returns. There are many situations in practice where controlling the returns acceptance can result in considerable cost savings, especially when the costs related to accepting a return are high. These costs may include, among others, transportation costs (related to the collection of returns), stock keeping costs, and recovery costs.

In this paper, we consider the hybrid system shown in Fig. 1. When a product is returned, it can be either rejected, or accepted and placed in a remanufacturable inventory, where it is assumed that in principle all accepted returns can be remanufactured. The finished good inventory can be replenished by manufacturing new products or remanufacturing accepted returns.

Manufacturing, remanufacturing as well as returns acceptance decisions can be based on different data. Two important data in this context are the finished good inventory position $(I)$ and the remanufacturable inventory position $(R)$. More precisely, I denotes

\footnotetext{
* Corresponding author. Tel.: +33476574746.

E-mail address: jean-philippe.gayon@grenoble-inp.fr (J.-P. Gayon).
}

the number of products in the finished good inventory plus the products actually being manufactured or remanufactured minus backlogs and $R$ denotes the number of products in the stock of accepted returned products not yet remanufactured.

We address the problem of jointly controlling manufacturing, remanufacturing, and returns acceptance control in a setting with stochastic processing times and finite capacities. The structure of the optimal policy is characterized helping us to design simple heuristic control rules for manufacturing, remanufacturing and returns acceptance. In a numerical study, we compare these heuristic control rules with the optimal rules.

The setup of the rest of the paper is as follows. Section 2 provides a literature review and points out our contributions to the literature and practice. In Section 3 the assumptions of our queueing control model are detailed. The structure of the optimal policy is derived in Section 4. In Section 5 we present several heuristic control rules for manufacturing, remanufacturing and returns acceptance. Then we compare them numerically with the optimal policy. The paper ends with a brief summary of the main results.

\section{Literature review}

The literature review focuses on the setting of Fig. 1 with two distinguished inventories: the remanufacturable inventory and the finished good inventory. We do not review papers where the 


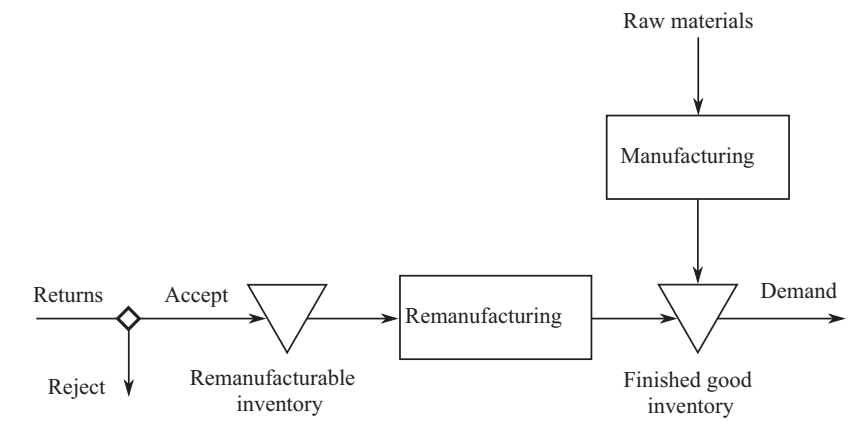

Fig. 1. Hybrid system with manufacturing, remanufacturing and returns acceptance.

remanufacturable inventory is not modelled explicitly. For instance Fleischmann et al. (2002) assume that returns can be re-used immediately as new products. In such a situation, the problem can usually be reduced to a single-dimensional problem where decisions are based only on the finished good inventory position. We also restrict our survey to papers assuming stochastic demands and returns. For exhaustive reviews, we refer the reader to Rubio et al. (2008) and Ilgin and Gupta (2010).

We begin with papers that investigate the structure of the optimal policy. In a periodic-review setting, Inderfurth (1997) studies a problem where returns can be accepted or rejected and unsatisfied demand is backlogged. When the manufacturing time and the remanufacturing time are equal (and constant), he proves the optimality of an $\left(S_{m}, S_{r}, S_{a}\right)$ policy with $S_{m}$ the manufacture up to level, $S_{r}$ the remanufacture up to level, and $S_{a}$ the accept (returns) up to level. The manufacturing decision and the acceptance decision are based on the aggregate inventory $(I+R)$ while the remanufacturing decision is based only on the inventory position $I$. More precisely, this policy states to manufacture if and only if (iff) $I+R<S_{m}$, remanufacture iff $I<S_{r}$ and accept returns iff $I+R<S_{a}$. To emphasize the link between decisions and data, we denote this policy by $\left(S_{m}[I+R], S_{r}[I], S_{a}[I+R]\right)$. We will use similar notations in the rest of this section. If the procurement time exceeds the remanufacturing time by one period, Inderfurth (1997) characterizes the optimal policy for the special case where the accepted returns are remanufactured directly without waiting. For systems with a remanufacturable inventory and non-identical manufacturing and remanufacturing times, the optimal policy has not yet been characterized. Before Inderfurth, Simpson (1978) had characterized the optimal policy for the case with zero manufacturing and remanufacturing times.

Simpson (1978) and Inderfurth (1997) consider a situation where they allow to dispose accepted return from the remanufacturable inventory. For both models, the optimal policy structure shows that the disposal option is only used when the returns arrive in the stock and not later. This makes these models equivalent to our model with respect to returns acceptance control.

Li et al. (2010) generalize the results of Simpson (1978) by including fixed manufacturing costs and fixed disposal costs. The optimal policy orders a quantity $S_{m}-I-R$ when $I+R$ drops below the reorder point $s_{m}$ and disposes $I+R-s_{a}$ returns (or at least $R$ returns if $I+R-s_{a}<0$ ) when $I+R$ rises above the disposal point $s_{a}$. DeCroix (2006) extends the results of Inderfurth (1997) to a multi-stage serial system where products are remanufactured at the upstream stage.

In what follows, we review papers focussing on heuristic policies. Kiesmüller (2003) investigates the problem with nonequal manufacturing and remanufacturing times. She proposes two heuristic policies assuming that all returns are accepted that we can denote by $\left(S_{m}\left[I^{\prime}\right], S_{r}[I]\right)$ and $\left(S_{m}[I+R], S_{r}\left[I^{\prime}\right]\right)$. She defines a modified inventory position $I^{\prime}$ that takes into account only part of the products being actually manufactured.

In a continuous-time review setting, van der Laan and Teunter (2006) assume that demand and returns occur according to independent Poisson processes. They include setup costs for manufacturing and remanufacturing. The times for manufacturing and remanufacturing are assumed to be equal. They investigate two heuristic policies where all returns are accepted. The $\left(s_{m}[I], Q_{m}, Q_{r}\right)$ policy orders a quantity $Q_{m}$ when the inventory position $I$ drops below the reorder point $s_{m}$. The remanufacturing is controlled by a push policy: as soon as there are $Q_{r}$ products in the remanufacturable stock, these products are sent to remanufacturing. The authors compare this policy with a pull remanufacturing policy $\left(s_{m}[I], Q_{m}, s_{r}[I], Q_{r}\right)$, with $s_{r}$ the reorder point for remanufacturing products. The authors provide approximate formulas for the optimal values of the different parameters and compare them to the optimal parameter values in a numerical study.

Van der Laan et al. (1996b) study a model with the option of rejecting returns upon arrival. The manufacturing time is constant while remanufacturing is operated by a finite number of servers with exponentially distributed times. There is a setup cost for manufacturing and no setup cost for remanufacturing. The authors propose an $\left(s_{m}[I+R], Q_{m}, S_{a}[R]\right)$ push remanufacturing policy and derive an analytical expression for the average cost. Van der Laan et al. (1996a) generalize the above policy via an $\left(s_{m}[I+R], Q_{m}, S_{a}^{1}[I+R], S_{a}^{2}[R]\right)$ push remanufacturing policy. For a system with remanufacturable stock holding cost, returns are accepted if both $I+R<S_{a}^{1}$, and $R<S_{a}^{2}$ hold. This returns acceptance policy resembles the Kanban generalized policy proposed by Liberopoulos and Dallery (2003).

Van der Laan and Salomon (1997) consider a model with correlated demand and return processes, demand and return inter-occurrence times being Coxian-2 distributed. The authors compare the $\left(s_{m}[I], Q_{m}, Q_{r}, S_{a}[I]\right)$ push remanufacturing policy with the $\left(S_{m}[I], Q_{m}, S_{r}[I], S_{r}, S_{a}[R]\right)$ pull remanufacturing policy, where the system remanufactures $S_{r}-I$ products if $I \leq S_{r}$. Teunter and Vlachos (2002) complement the numerical study of this model. In a multi-echelon setting, Aras et al. (2006) consider a two stage problem with remanufacturing at the downstream stage.

Korugan and Gupta (2000) have investigated very briefly the same setting as ours, proposing a Kanban policy. However, neither theoretical results nor numerical results are presented in this work.

We now summarize our contributions with respect to the literature. Our first contribution is the characterization of the optimal policy for the setting described in Section 1. We prove that the optimal policy, minimizing discounted or average costs, is characterized by two state-dependent base-stock thresholds for manufacturing and remanufacturing and one state-dependent returns acceptance threshold. We also derive several monotonicity results for these thresholds. To the best of our knowledge, only Simpson (1978), Inderfurth (1997), and Li et al. (2010) have derived optimality results for the setting of Fig. 1. However, they assume constant times and infinite capacities for manufacturing and remanufacturing while we assume stochastic processing times and finite capacities. Our second contribution is the comparison of heuristic policies with the optimal policy. We restrict our attention to heuristics that are consistent with our theoretical monotonicity results. Most of these heuristic policies have been studied in the literature in different contexts but have not been compared with the optimal policy. Designing from scratch efficient heuristics that jointly control manufacturing, remanufacturing and acceptance is a difficult task. To deal with this difficulty, we first consider heuristic policies where only one of the three controls (manufacturing or remanufacturing or returns acceptance) is a heuristic and the two other controls are set optimally given this heuristic control. It allows us to derive insights about the relevance of various heuristics for the three types of control. Based on this analysis, we derive insights on several heuristics that jointly control manufacturing, remanufacturing and acceptance. 
We do not include setup times, setup costs and disposal options. Setup times and costs can be neglected typically when manufacturing and remanufacturing require the same mindset of the person executing both activities, as well as the same tools, same materials. We also assume that preemption is possible. Hereafter we give an example where the above assumptions fit quite well. Companies like Dell build tailor-made products which may for instance be returned as commercial returns. The configuration of a return may (almost) correspond with the configuration of a demanded product. Then, especially in case of very expensive components, it can be better to start with the return instead of continuing building new from scratch. Or vice versa, when e.g. the time to reconfigure a returned product takes far more time than starting new from scratch.

\section{Problem formulation}

We consider a single item production-inventory hybrid system where product demand can be fulfilled either by manufacturing new products or by remanufacturing returned products (see Fig. 2).

Manufacturing and remanufacturing are modelled by single servers $M_{m}$ and $M_{r}$ with exponentially distributed processing times (rates $\mu_{m}$ and $\mu_{r}$ ). These two servers can start or stop processing at any time (preemption is allowed). The unit manufacturing and remanufacturing costs are respectively denoted by $c^{m}$ and $c^{r}$. Returns arrive according to a Poisson process with rate $\delta$ independent of the demand process. They can be either rejected upon arrival with cost $c^{b}$ or accepted with $\operatorname{cost} c^{a}$. It is not allowed to dispose a return once accepted. When a return is accepted, it is placed in a remanufacturable product inventory $B_{1}$. Via the remanufacturing server, remanufacturable products can be moved to the finished good inventory $B_{2}$. In parallel, the manufacturing server can also fill the finished good inventory $B_{2}$. Inventory $B_{2}$ sees customer demand arriving according to a Poisson process with rate $\lambda$. Customer demand can be satisfied as well by new or remanufactured products. We assume that backorders are allowed and there are no storage restrictions. At time $t$, the net inventory level at $B_{2}$ (resp. $B_{1}$ ) is denoted by $X_{2}(t)$ (resp. $X_{1}(t)$ ). Note that $X_{2}(t)$ can be negative due to backorders. When buffer $B_{1}$ is empty, remanufacturing is not possible. Per unit of time, the system incurs in state $\mathbf{x}=\left(x_{1}, x_{2}\right)$ a cost rate $C(\mathbf{x})=h_{1} x_{1}+h_{2} x_{2}^{+}+b x_{2}^{-}$where $h_{i}$ is the unit inventory holding cost at buffer $B_{i}, b$ is the unit backlog cost, $x^{+}=\max \{0, x\}$ and $x^{-}=-\min \{0, x\}$. To ensure the stability of the system, we have to assume that the demand rate is smaller than the total production capacity rate: $\lambda<\mu_{m}+\min \left\{\mu_{r}, \delta\right\}$.

A control policy $\pi=\left(\pi_{a}, \pi_{r}, \pi_{m}\right)$ consists of three control rules: the returns acceptance control rule $\pi_{a}$, the remanufacturing control rule $\pi_{\mathrm{r}}$, and the manufacturing control rule $\pi_{m}$. The returns acceptance control rule (respectively manufacturing control rule, remanufacturing control rule) specifies when to accept returned products (respectively when to produce new items, when to remanufacture returned products). In the rest of the paper, a control policy will be called for short a policy.

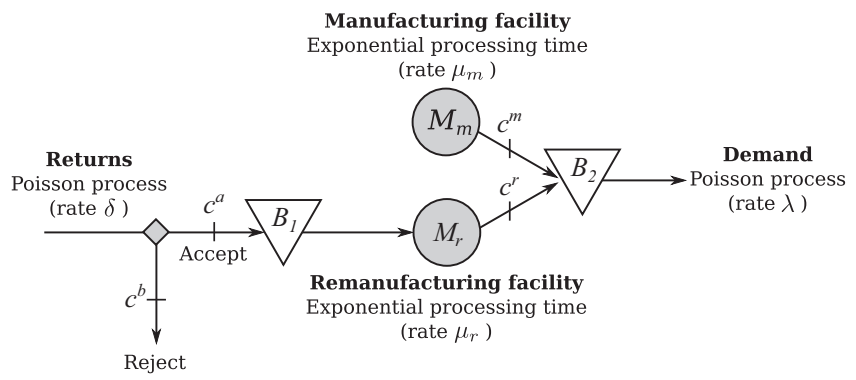

Fig. 2. Model of the hybrid system with manufacturing, remanufacturing and returns acceptance.
The discounted expected cost over an infinite horizon for a policy $\pi$, with $\mathbf{x}=\left(x_{1}, x_{2}\right)$ the state of the system when $t=0$ and $\alpha>0$ the discount rate, is given by

$v^{\pi}(\mathbf{x})=E_{\mathbf{x}}^{\pi}\left[\int_{0}^{+\infty} e^{-\alpha t} C(\mathbf{X}(t)) \mathrm{d} t\right]+E_{\mathbf{x}}^{\pi}\left[\sum_{i=1}^{\infty}\left(\begin{array}{c}e^{-\alpha \phi_{a}^{(i)}} c^{a}+e^{-\alpha \phi_{b}(i)} c^{b} \\ +e^{-\alpha \phi_{m}(i)} c^{m}+e^{-\alpha \phi_{r}(i)} c^{r}\end{array}\right)\right]$

where $\phi_{a}(i), \phi_{b}(i), \phi_{m}(i)$ and $\phi_{r}(i)$ respectively represent the $i$ th event time when either a return is accepted, a return is rejected, a product is manufactured or an accepted return is remanufactured. The objective is to minimize the expected discounted cost over an infinite horizon. Let $v^{\star}$ be the optimal value function defined by

$v^{\star}\left(x_{1}, x_{2}\right)=\min _{\pi}\left\{v^{\pi}\left(x_{1}, x_{2}\right)\right\}$.

The optimal policy is denoted by $\pi^{\star}=\left(\pi_{a}^{\star}, \pi_{r}^{\star}, \pi_{m}^{\star}\right)$.

We are also interested in the average cost problem over an infinite horizon. The theoretical results derived in the next section for the discounted cost problem pertains to the average cost problem (Puterman, 1994).

\section{Characterization of the optimal policy}

The discounted cost problem can be formulated as a continuous time Markov Decision Process (MDP). After uniformization, rate $\tau=\lambda+\mu_{r}+\mu_{m}+\delta+\alpha$, we can transform the continuous time MDP into a discrete time MDP (Puterman, 1994). The optimal value function satisfies the following optimality equations:

$v^{\star}=\mathcal{T} v^{\star}$,

with

$\mathcal{T} v\left(x_{1}, x_{2}\right)=\frac{1}{\tau}\left(\begin{array}{c}C\left(x_{1}, x_{2}\right)+\lambda v\left(x_{1}, x_{2}-1\right) \\ +\delta T_{a} v\left(x_{1}, x_{2}\right)+\mu_{r} T_{r} v\left(x_{1}, x_{2}\right) \\ +\mu_{m} T_{m} v\left(x_{1}, x_{2}\right)\end{array}\right)$,

and

$T_{a} v\left(x_{1}, x_{2}\right)=\min \left\{v\left(x_{1}, x_{2}\right)+c^{b}, v\left(x_{1}+1, x_{2}\right)+c^{a}\right\}$,

$T_{m} v\left(x_{1}, x_{2}\right)=\min \left\{v\left(x_{1}, x_{2}\right), v\left(x_{1}, x_{2}+1\right)+c^{m}\right\}$,

$T_{r} v\left(x_{1}, x_{2}\right)= \begin{cases}v\left(x_{1}, x_{2}\right) & \text { if } x_{1}=0, \\ \min \left\{v\left(x_{1}, x_{2}\right), v\left(x_{1}-1, x_{2}+1\right)+c^{r}\right\} & \text { if } x_{1}>0 .\end{cases}$

The operators $T_{a}, T_{r}$ and $T_{m}$ are related to respectively the returns acceptance control, the remanufacturing control and the manufacturing control.

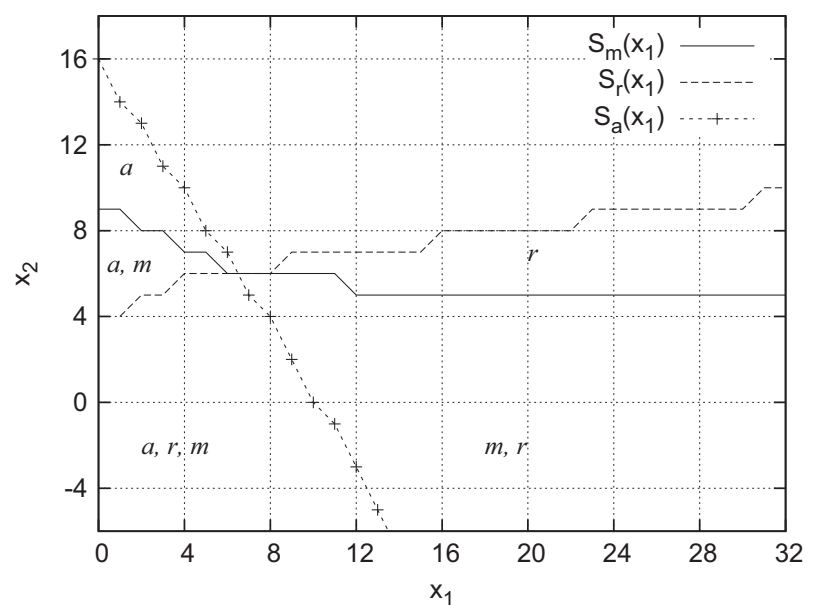

Fig. 3. Average cost optimal policy ( $\delta=0.6, \mu_{r}=0.6, \mu_{m}=0.6, \lambda=1, h_{1}=1, h_{2}=5$ $\left.b=10, c^{a}=c^{r}=c^{m}=c^{b}=0\right)$, with $a, m, r$ respectively denoting the areas where the system accepts returns, manufactures, and remanufactures products. 
In order to prove that the optimal policy has some structural properties, we will show that the optimal value function is supermodular and superconvex (Koole, 1998). Let $V$ be a set of realvalued functions in $\mathbb{N} \times \mathbb{Z}$ with the following properties.

Definition 1. If $v \in V$, then for all $\left(x_{1}, x_{2}\right) \in \mathbb{N} \times \mathbb{Z}$

(a) $v$ is supermodular:

$v\left(x_{1}+1, x_{2}+1\right)-v\left(x_{1}+1, x_{2}\right)$

$-v\left(x_{1}, x_{2}+1\right)+v\left(x_{1}, x_{2}\right) \geq 0$,

(b) $v$ is superconvex:

$$
\left\{\begin{array}{c}
v\left(x_{1}+2, x_{2}\right)-v\left(x_{1}+1, x_{2}+1\right) \\
-v\left(x_{1}+1, x_{2}\right)+v\left(x_{1}, x_{2}+1\right) \geq 0, \text { and } \\
v\left(x_{1}, x_{2}+2\right)-v\left(x_{1}+1, x_{2}+1\right) \\
-v\left(x_{1}, x_{2}+1\right)+v\left(x_{1}+1, x_{2}\right) \geq 0
\end{array}\right.
$$

Note that supermodularity and superconvexity imply together convexity in directions $x_{1}$ and $x_{2}$ :

$\left\{\begin{array}{l}v\left(x_{1}+2, x_{2}\right)-2 v\left(x_{1}+1, x_{2}\right)+v\left(x_{1}, x_{2}\right) \geq 0, \text { and } \\ v\left(x_{1}, x_{2}+2\right)-2 v\left(x_{1}, x_{2}+1\right)+v\left(x_{1}, x_{2}\right) \geq 0 .\end{array}\right.$

We show in Appendix A that if a value function $v$ is in $V$ then $\mathcal{T} v$ is also in $v$. As operator $\mathcal{T}$ is a contraction mapping, it implies the optimality equations (1) that the optimal value function $v^{*}$ is also in $V$. We are now ready to state our main result on the structure of the optimal policy (see Appendix A for a detailed proof).

Theorem 1. The optimal value function $v^{*}$ belongs to V. Further, the optimal policy $\pi^{\star}$ consists of two state-dependent base-stock levels $S_{m}\left(x_{1}\right)$ and $S_{r}\left(x_{1}\right)$ for manufacturing and remanufacturing and one state-dependent return acceptance threshold $S_{a}\left(x_{1}\right)$ such that

- Produce at $M_{m}$ iff $x_{2}<S_{m}\left(x_{1}\right)$. Moreover $S_{m}\left(x_{1}\right)-1 \leq S_{m}\left(x_{1}+1\right)$ $\leq S_{m}\left(x_{1}\right)$.

- Remanufacture at $M_{r}$ iff $x_{2}<S_{r}\left(x_{1}\right)$. Moreover $S_{r}\left(x_{1}\right) \leq S_{r}\left(x_{1}+1\right)$.

- Accept returns in $B_{1}$ iff $x_{2}<S_{a}\left(x_{1}\right)$. Moreover $S_{a}\left(x_{1}+1\right) \leq S_{a}$ $\left(x_{1}\right)-1$.

So the optimal policy consists of three switching curves. Each switching curve divides the state space into two and is associated with one decision to take. To illustrate Theorem 1, we provide in Fig. 3 the switching curves as a function of the state of the system for one set of parameter values. These curves are obtained with a value iteration algorithm based on Bellman equation (1). More details on the computational procedure are given in Appendix D. The properties of the switching curves illustrated in Fig. 3 will be used in Section 5 to build simple threshold policies that approximate the optimal policy.

When $h_{1} \geq h_{2}$, we can derive the following additional intuitive result which states that a push remanufacturing policy is optimal when it is more expensive to keep in stock remanufacturable products rather than finished good products. The proof can be found in Appendix B.

Theorem 2. If $h_{1} \geq h_{2}$, a push remanufacturing policy is optimal (remanufacture whenever $x_{1}>0$ ).

\section{Heuristic policies}

The optimal policy $\pi^{\star}=\left(\pi_{a}^{\star}, \pi_{r}^{\star}, \pi_{m}^{\star}\right)$ consists of three control rules: the returns acceptance control rule $\pi_{a}^{*}$, the remanufacturing control rule $\pi_{r}^{*}$, and the manufacturing control rule $\pi_{m}^{*}$. Each optimal control rule can be summarized by a rather complex switching curve which delimitates when to manufacture or not, when to remanufacture or not and when to accept returns or not (see Theorem 1 and Fig. 3). In the literature, simple heuristic threshold control rules are used instead of these complex switching curves.

The main purpose of this section is to compare the optimal policy $\pi^{\star}$ to the heuristic policies used in the literature. If heuristic control rules are used for the three decisions (manufacturing, remanufacturing and returns acceptance), it is difficult to explain the gap with the optimal policy. In order to isolate the effect of one heuristic control rule on the optimality gap, we introduce policy $\pi^{\star}\left(\pi_{m}\right)$ which is the best policy given that manufacturing control rule $\pi_{m}$ is applied. For instance, $\pi_{m}$ can consist in manufacturing new products when the serviceable inventory is smaller than some threshold. Similarly we can define respectively $\pi^{\star}\left(\pi_{r}\right)$ and $\pi^{\star}\left(\pi_{a}\right)$ as the best policies given that remanufacturing control rule $\pi_{\mathrm{r}}$ and returns acceptance control rule $\pi_{a}$ are applied. In a second step, we study joint heuristic policies where the three rules (manufacturing, remanufacturing and returns acceptance) are heuristic rules.

\subsection{Set up of the numerical experiments}

Our numerical results are based on 6160 instances being the combinations of

$\alpha \in\{0.1,0\}, \quad \lambda=1, \quad \delta \in\{0.2,0.5,0.8,1.1\}$,

$\mu_{r} \in\{0.2,0.5,1,2\}, \quad \mu_{m} \in\{0.2,0.5,1,2\}$,

$c^{b}=c^{r}=0, \quad c^{m} \in\{0,5,10\}, \quad c^{a} \in\{0,5,10\}$,

$h_{1}=1, \quad h_{2} \in\{1.5,5,10\}$,

$b \in\{2,10,100\}$,

that satisfy the stability condition $\lambda<\mu_{m}+\min \left\{\mu_{r}, \delta\right\}$. We explain in Appendix $C$ how to relax easily $c^{b}=c^{r}=0, \lambda=1$ and $h_{1}=1$ for teh average cost problem.

For each of these instances, we compute the average cost $(\alpha=0)$ and the discounted cost $(\alpha=0.1)$ for the optimal policy and for various heuristic policies. Our computational procedure is detailed in Appendix D. We denote by $g(\pi)$ the average cost (respectively discounted cost) of a policy $\pi$ when $\alpha=0$ (respectively $\alpha=0.1$ ). In the discounted cost case, we compute the value functions when the initial state is $\mathbf{x}=(0,0)$. The deviation between a policy $\boldsymbol{\pi}$ and the optimal policy $\boldsymbol{\pi}^{*}$ is denoted by

$\Delta g(\boldsymbol{\pi})=\frac{g(\boldsymbol{\pi})-g\left(\boldsymbol{\pi}^{\star}\right)}{g\left(\boldsymbol{\pi}^{\star}\right)}$.

The quantity $\Delta g(\pi)$ represents the percentage cost increase when using policy $\pi$ instead of the optimal policy $\pi^{*}$. For a given policy $\pi$, we denote the average deviation over the 6160 instances by $\overline{\Delta g(\boldsymbol{\pi})}$, the maximal deviation by $\max \{\Delta g(\boldsymbol{\pi})\}$, and the percentage of instances with a deviation lower than $\beta \%$ by $\Delta g(\boldsymbol{\pi})<\beta \%$.

\subsection{Manufacturing control rules}

From Theorem 1 the optimal manufacturing switching curve $S_{m}\left(x_{1}\right)$ has a slope between 0 and $-45^{\circ}$ (see Fig. 4). The two extreme cases are the horizontal switching curve and the decreasing diagonal switching curve. We limit our attention to manufacturing control rules that are consistent with the optimal manufacturing rule.

In the following description of the heuristic manufacturing control rules, $Z_{m}$ denotes a policy parameter:

$\left[x_{2}\right]_{m}$ : Manufacture iff $x_{2}<Z_{m}$ (see Fig. 5a). $\left[x_{1}+x_{2}\right]_{m}$ : Manufacture iff $x_{1}+x_{2}<Z_{m}$ (see Fig. 5b). 


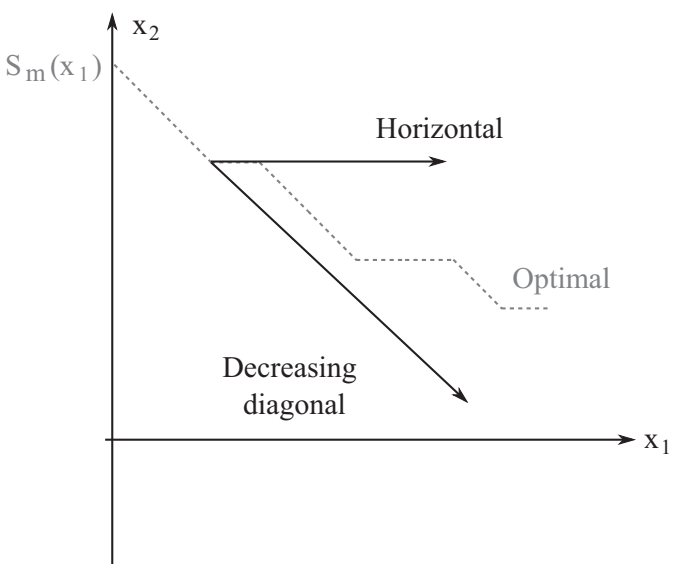

Fig. 4. Slope of the optimal manufacturing switching curve.
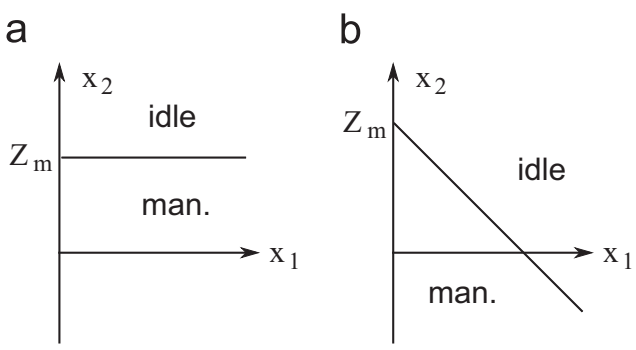

Fig. 5. Heuristic manufacturing control rules. (a) $\left[x_{2}\right]_{m}$. (b) $\left[x_{1}+x_{2}\right]_{m}$.

For a given $Z_{m}$, we compute the optimal policy by dynamic programming and then we optimize over all possible values of $Z_{m}$ (see Appendix D for details).

Manufacturing control rules $\left[x_{2}\right]_{m}$ and $\left[x_{1}+x_{2}\right]_{m}$ have been studied respectively in van der Laan and Salomon (1997), Korugan and Gupta (2000), van der Laan and Teunter (2006) and in Simpson (1978), Inderfurth (1997), van der Laan et al. (1996a, 1996b), Kiesmüller (2003).

Table 1 provides a summary of our extensive numerical study comparing the different manufacturing control rules with the optimal manufacturing control rule. We observe that optimally controlling manufacturing can make a significant difference in some cases. One instance has a cost deviation greater than $6 \%$ for the two heuristic manufacturing control policies.

However, the $\left[x_{1}\right]_{m}$ and $\left[x_{1}+x_{2}\right]_{m}$ control rules have excellent average performances. The main criterion for choosing between $\left[x_{2}\right]_{m}$ and $\left[x_{1}+x_{2}\right]_{m}$ is the ratio $\mu_{r}$ to $\mu_{m}$. In Fig. 6 (and other numerical experiments not reported) we observe that $\left[x_{1}+x_{2}\right]_{m}$ is near optimal when the remanufacturing rate $\mu_{r}$ is large relative to the manufacturing rate $\mu_{m}$. If $\mu_{r}$ is large, a remanufacturable product can be available quickly to serve demand, so it is logical to control manufacturing with the aggregate inventory level $\left(x_{1}+x_{2}\right)$. Inversely, when $\mu_{r}$ is small, a remanufacturable product cannot be available to serve demand before a long time, so the manufacturing control rule should be based on $\left[x_{2}\right]_{m}$. Our observations are consistent with Simpson (1978) and Inderfurth (1997) who proves that controlling manufacturing with $\left[x_{1}+x_{2}\right]_{m}$ is optimal when manufacturing and remanufacturing times are constant and equal and when there is no capacity constraint.

The main insights derived in this section can be summarized as follows:

- Implementing the optimal manufacturing control rule can lead to large cost reductions in comparison with the heuristic control rules $\left[x_{2}\right]_{m}$ and $\left[x_{1}+x_{2}\right]_{m}$. Moreover, there exist instances for which the two heuristic manufacturing control rules have poor performances.
Table 1

Comparison of the heuristic manufacturing control rules with to the optimal rule.

\begin{tabular}{llll}
\hline$\pi$ & $\overline{\Delta g(\pi)}(\%)$ & $\max \{\Delta g(\pi)\}(\%)$ & $\Delta g(\pi)<1 \%(\%)$ \\
\hline$\pi^{\star}\left(\left[x_{2}\right]_{m}\right)$ & 0.39 & 9.82 & 87.9 \\
$\pi^{\star}\left(\left[x_{1}+x_{2}\right]_{m}\right)$ & 1.03 & 23.7 & 69.9 \\
\hline
\end{tabular}

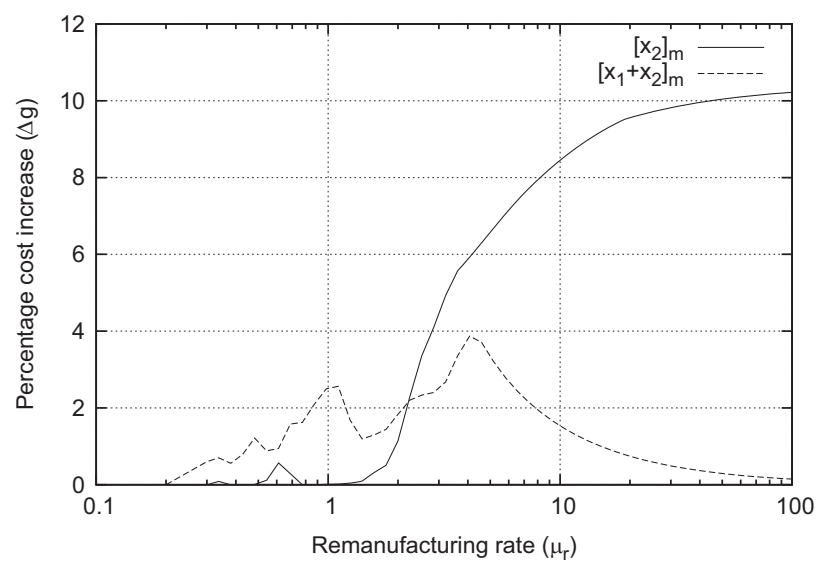

Fig. 6. The effect of $\mu_{r}$ on the performances of the manufacturing control rules $\left(\delta=0.8, \mu_{m}=1, \lambda=1, h_{1}=1, h_{2}=4, b=5, c^{a}=c^{r}=c^{m}=c^{b}=0, \alpha=0\right)$.

- The manufacturing control rule $\left[x_{2}\right]_{m}$ that uses only local data is nearly optimal when the remanufacturing time is large.

- The manufacturing control rule $\left[x_{1}+x_{2}\right]_{m}$ that uses two types of data (the remanufacturable inventory level and the finished good inventory level) performs well when the remanufacturing time is small.

\subsection{Remanufacturing control rules}

As in the previous section, we focus on remanufacturing control rules that are consistent with the structure of the optimal policy established in Theorem 1 and illustrated in Fig. 7. The two extreme cases are the horizontal switching curve and the vertical switching curve.

In the following description of the heuristic remanufacturing control rules, we denote by $Z_{r}$ a remanufacturing control rule parameter that will be optimized in the numerical study:

$\left[x_{2}\right]_{r}$ : Remanufacture iff $x_{2}<Z_{r}$ (see Fig. 8a).

$\left[x_{1}\right]_{r}$ : Remanufacture iff $x_{1}>Z_{r}$ (see Fig. 8b).

$[p u s h]_{r}$ : Remanufacture whenever possible, i.e. when $x_{1}>0$ (see Fig. 8c).

Control rules $\left[x_{2}\right]_{r}$ and $[p u s h]_{r}$ have been respectively studied in Simpson (1978), Inderfurth (1997), van der Laan and Salomon (1997), Korugan and Gupta (2000), van der Laan and Teunter (2006), Kiesmüller (2003) and in van der Laan et al. (1996a,b), van der Laan and Salomon (1997), van der Laan and Teunter (2006). As far as we know, control rule $\left[x_{1}\right]_{r}$ has not been mentioned in the literature before.

The extensive numerical study shows that the $\left[x_{2}\right]_{r}$ control rule performs extremely well with an average deviation of $0.06 \%$ and a worst-case deviation of $3.41 \%$ (see Table 2). The $[p u s h]_{r}$ and the $\left[x_{1}\right]_{r}$ control rules have exactly the same performances. This might look surprising but there is a simple explanation for this. The $[p u s h]_{r}$ control rule is a special case of the $\left[x_{1}\right]_{r}$ control rule with $Z_{r}=0$. One can easily show that the optimal $Z_{r}$ for $\left[x_{1}\right]_{r}$ is precisely 0 and the two control rules are then equivalent. 


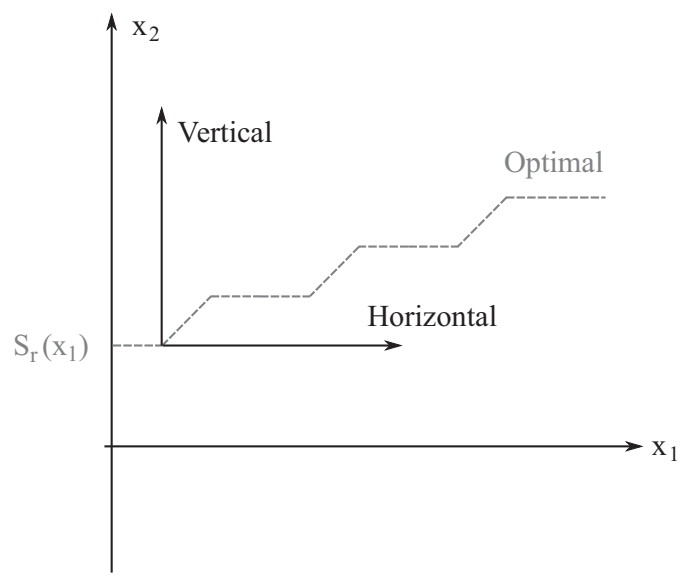

Fig. 7. Slope of the optimal remanufacturing switching curve.

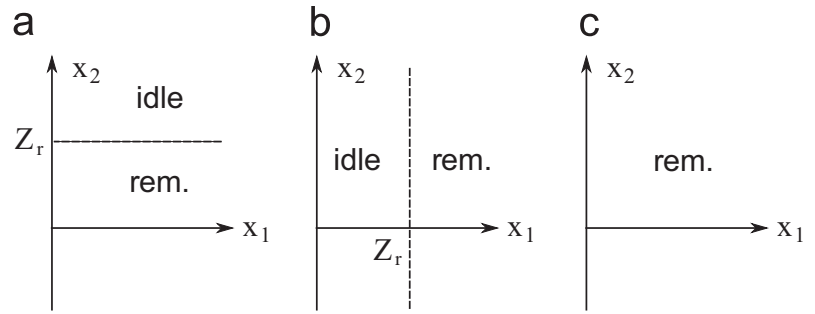

Fig. 8. Heuristic remanufacturing control rules. (a) $\left[x_{2}\right]_{r}$. (b) $\left[x_{1}\right]_{r}$. (c) $[p u s h]_{r}$.

Table 2

Comparison of heuristic remanufacturing control rules to the optimal.

\begin{tabular}{llll}
\hline$\pi$ & $\overline{\Delta g(\pi)}(\%)$ & $\max \{\Delta g(\pi)\}(\%)$ & $\Delta g(\pi)<1 \%(\%)$ \\
\hline$\pi^{\star}\left(\left[x_{2}\right]_{r}\right)$ & 0.06 & 3.41 & 99.0 \\
$\pi^{\star}\left(\left[x_{1}\right]_{r}\right)$ & 4.70 & 135 & 59.4 \\
$\pi^{\star}\left([\text { push }]_{r}\right)$ & 4.70 & 135 & 59.4 \\
\hline
\end{tabular}

When $h_{1} \geq h_{2}$, the [push $]_{r}$ control rule is optimal (see Theorem 2). As the $[p u s h]_{r}$ control rule is a special case of the $\left[x_{2}\right]_{r}$ control rule, the $\left[x_{2}\right]_{r}$ control rule is also optimal when $h_{1} \geq h_{2}$. Fig. 9 plots the effect of ratio $h_{1}$ on the average costs. We observe that the $\left[x_{2}\right]_{r}$ control rule is always nearly optimal. We also see that the $[p u s h]_{r}$ control rule can have a poor performance when $h_{1}<h_{2}$.

The main insights derived in this section can be summarized as follows:

- Controlling remanufacturing with only the finished good inventory data is sufficient since the $\left[x_{2}\right]_{r}$ control rule has extremely good performances.

- The $[p u s h]_{r}$ control rule is optimal when $h_{1} \geq h_{2}$ and is near optimal when $h_{1}$ close to $h_{2}$. Otherwise, it might have very poor performances.

\subsection{Returns acceptance control rules}

The form of the optimal returns acceptance switching curve according to Theorem 1 is sketched in Fig. 10. The two extreme cases are the decreasing diagonal switching curve and the vertical switching curve.

In the following description, $Z_{a}$ is a returns acceptance control rule parameter that will be optimized in the numerical study:

$\left[x_{1}+x_{2}\right]_{a}$ : Accept returns iff $x_{1}+x_{2}<Z_{a}$ (see Fig. 11a). $\left[x_{1}+x_{2}^{+}\right]_{a}$ : Accept returns iff $x_{1}+x_{2}^{+}<Z_{a}$ (see Fig. 11b).

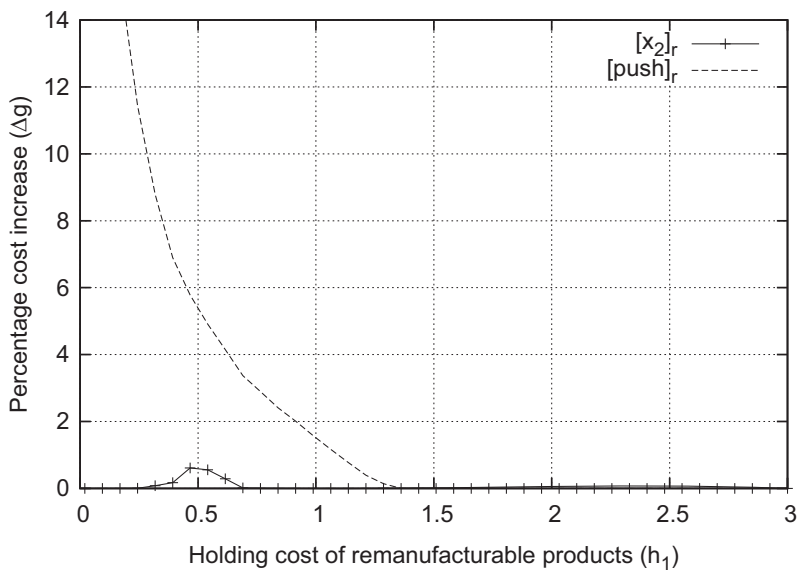

Fig. 9. The effect of $h_{1}$ on the performances of the manufacturing control rules $\left(\delta=0.8, \mu_{r}=\mu_{m}=\lambda=1, h_{2}=2, b=5, c^{a}=c^{r}=c^{m}=c^{b}=0, \alpha=0\right)$.

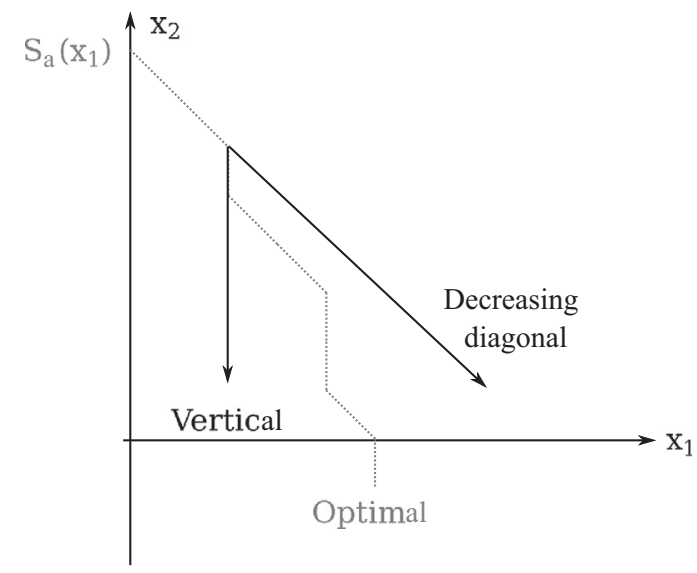

Fig. 10. Slope of the optimal returns acceptance switching curve.

$\left[x_{1}\right]_{a}$ : Accept returns iff $x_{1}<Z_{a}$ (see Fig. 11c).

$[a c c]_{a}$ : Accept all returns (see Fig. 11d).

$[r e j]_{a}$ : Reject all returns (see Fig. 11c).

Control rules $\left[x_{1}+x_{2}\right]_{a} ;\left[x_{1}+x_{2}^{+}\right]_{a} ;\left[x_{1}\right]_{a}$ and $[a c c]_{a}$ have respectively been investigated in Simpson (1978), Inderfurth (1997); Korugan and Gupta (2000); van der Laan et al. (1996b), van der Laan and Salomon (1997) and van der Laan and Teunter (2006), Kiesmüller (2003).

The control rules $[a c c]_{a}$ and $[r e j]_{a}$ have clearly the worst performances since they are special cases of the three other control rules. For instance $\left[x_{1}\right]_{a}$ is equivalent to $[a c c]_{a}$ when the policy parameter $Z_{a}$ goes to $\infty$ and to $[r e j]_{a}$ when $Z_{a}$ goes to $-\infty$.

Table 3 provides a summary of our numerical study comparing the acceptance control rules. We first observe that controlling optimally the returns acceptance can make a significant difference for each of the heuristic acceptance control rule. The five control rules have a worst-case performance larger than $17 \%$. The worstcase performance for control rules $[a c c]_{a}$ and $[r e j]_{a}$ is even not bounded, due to instability reasons. Under $[a c c]_{a}$, the remanufacturable inventory is unstable when the return rate $\delta$ exceeds the remanufacturing rate $\mu_{r}$. Under $[r e j]_{a}$, the backlog queue is unstable when the system is under-capacitated $\left(\lambda \geq \mu_{r}\right)$.

Among the 6160 instances, one instance has a percentage cost increase $\Delta g$ greater than $7 \%$ for the five returns acceptance control rules under consideration. So, we observe that optimally controlling the returns acceptance can make a significant difference. 
a

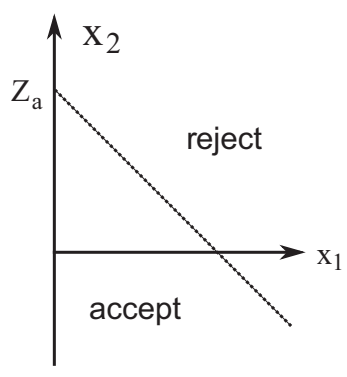

b

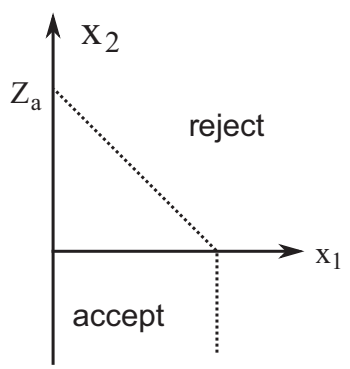

C

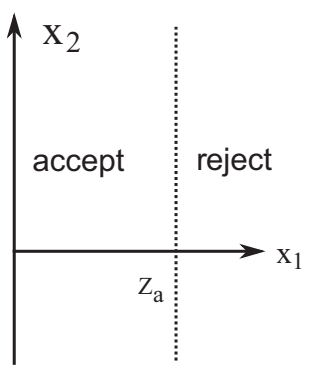

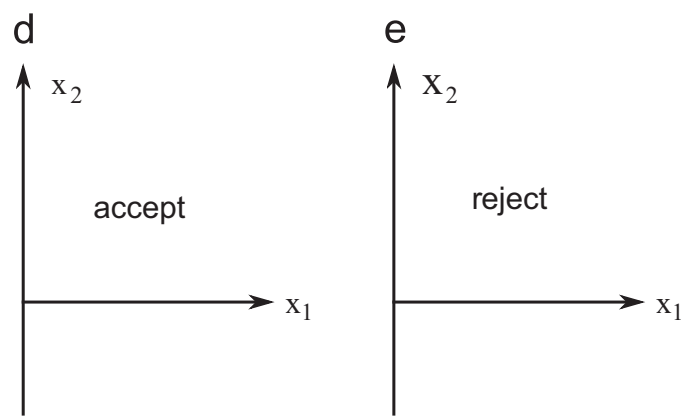

Fig. 11. Heuristic returns acceptance control rules. (a) $\left[x_{1}+x_{2}\right]_{a}$. (b) $\left[x_{1}+x_{2}^{+}\right]_{a}$. (c) $\left[x_{1}\right]_{a}$. (d) $[a c c]_{a}$. (e) $[r e j]_{a}$.

Table 3

Comparison of heuristic returns acceptance control rules with the optimal rule.

\begin{tabular}{llll}
\hline$\pi$ & $\overline{\Delta g(\pi)}$ & $\max \{\Delta g(\pi)\}$ & $\Delta g(\pi)<1 \%(\%)$ \\
\hline$\pi^{*}\left(\left[x_{1}+x_{2}\right]_{a}\right)$ & $1.00 \%$ & $26.4 \%$ & 76.7 \\
$\pi^{*}\left(\left[x_{1}+x_{2}^{+}\right]_{a}\right)$ & $1.02 \%$ & $17.6 \%$ & 71.9 \\
$\pi^{*}\left(\left[x_{1}\right]_{a}\right)$ & $1.75 \%$ & $25.3 \%$ & 53.6 \\
$\pi^{*}\left([r e j]_{a}\right)$ & $\infty$ & $\infty$ & 8.66 \\
$\pi^{*}\left([a c c]_{a}\right)$ & $\infty$ & $\infty$ & 13.5 \\
\hline
\end{tabular}

If we focus on control rules $\left[x_{1}+x_{2}\right]_{a},\left[x_{1}+x_{2}^{+}\right]_{a}$ and $\left[x_{1}\right]_{a}$, it is difficult to decide when to use one control rule or the other. This is illustrated in Fig. 12 which shows the non-monotonic effect of the remanufacturing rate on the deviation. In other numerical experiments (not reported here), we observe other behaviors. In all our numerical tests, we always observe that control rule $\left[x_{1}+x_{2}\right]_{a}$ should be preferred to $\left[x_{1}+x_{2}^{+}\right]_{a}$ when $\mu_{r}$ is large enough. When $\mu_{r}$ is large, stocks $B_{1}$ and $B_{2}$ can be merged into a virtual single stock which leads to good performances of the control rule $\left[x_{1}+x_{2}\right]_{a}$. When $\mu_{r}$ is small enough, $\left[x_{1}+x_{2}^{+}\right]_{a}$ outperforms $\left[x_{1}+x_{2}\right]_{a}$. In this case, the remanufacturing server is the bottleneck and it is useless to accept an additional return since the remanufacturing facility will not process it quickly enough. Hence, controlling returns with $\left[x_{1}+x_{2}^{+}\right]_{a}$ is more efficient since it does not take into account backlogs. For the same reasons, when $\mu_{r}$ is very small, the control rule $\left[x_{1}\right]_{a}$ may outperform other control rules. Note that control rule $\left[x_{1}\right]_{a}$ only considers local data for controlling returns acceptance.

The main insights derived in this section are

- For all heuristic returns acceptance control rules, the cost deviation from the optimal policy can be large and implementing the optimal manufacturing control can lead to large cost reductions.

- There exist instances for which all heuristic returns acceptance control rules have poor performances.

- For short remanufacturing times, the $\left[x_{1}+x_{2}\right]_{a}$ control rule should be preferred to $\left[x_{1}+x_{2}^{+}\right]_{a}$ and vice versa.

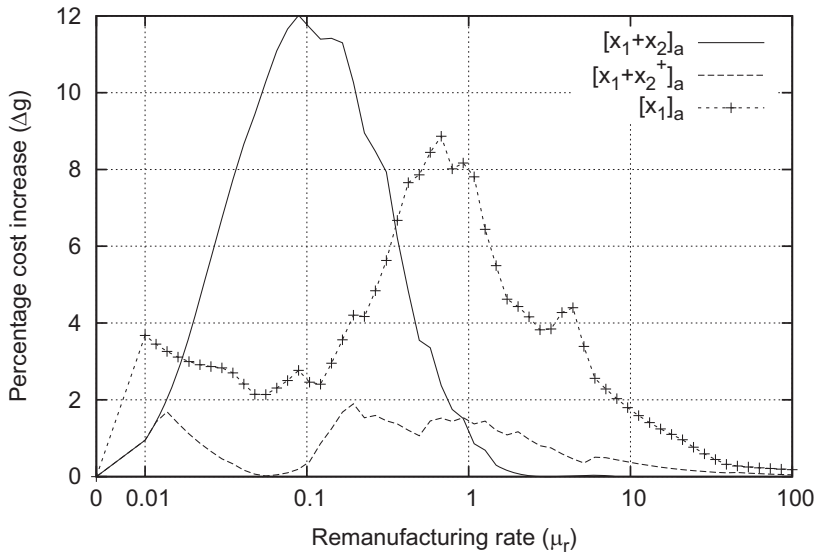

Fig. 12. The effect of $\mu_{r}$ on the performances of the returns acceptance control rules $\left(\delta=0.8, \mu_{m}=1.2, \lambda=1, h_{1}=1, h_{2}=2, b=5, c^{a}=c^{r}=c^{m}=c^{b}=0, \alpha=0\right)$.

- Control rule $\left[x_{1}\right]_{a}$ using only local data might have very good performances.

\subsection{Joint heuristic strategies}

In this section we consider heuristic policies that simultaneously use heuristic control rules for manufacturing, remanufacturing and returns acceptance. In line with our previous insights, we exclude control rules that have poor performances and focus on the following control rules:

- Manufacturing: $\left[x_{2}\right]_{m}$ and $\left[x_{1}+x_{2}\right]_{m}$.

- Remanufacturing: $\left[x_{2}\right]_{r}$.

- Returns acceptance: $\left[x_{1}+x_{2}\right]_{a},\left[x_{1}+x_{2}^{+}\right]_{a}$ and $\left[x_{1}\right]_{a}$.

In the literature (see Section 2) several heuristic policies can be seen as combinations of these control rules. We need to slightly adapt them to the situation studied in this paper. As we do not include setup costs, we set the lot size $Q$ to 1 . As preemption is allowed, we replace $I$ (the inventory position of finished goods) 

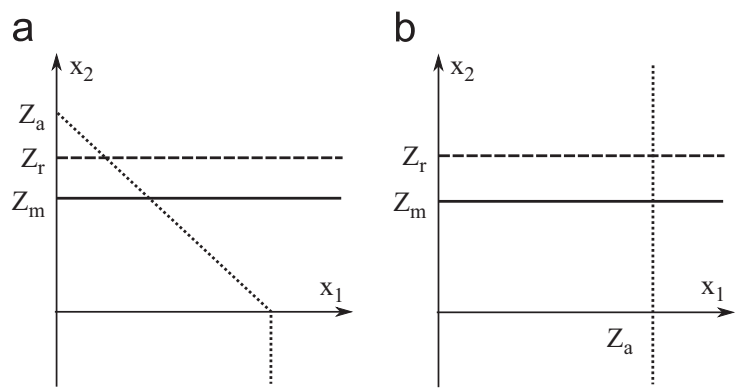

C

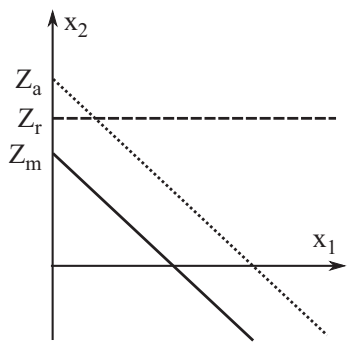

Fig. 13. Heuristic policies adapted from the literature. (a) KB. (b) FB. (c) BSE. a

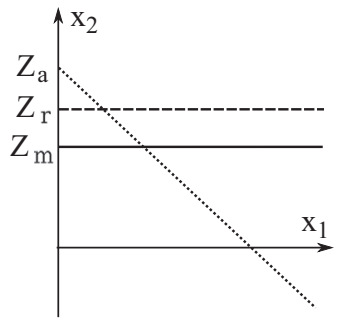

b

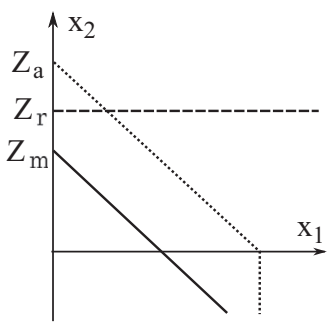

Fig. 14. New heuristic policies. (a) BSR. (b) KBR.

Table 4

Comparison of the heuristic policies with the optimal policy.

\begin{tabular}{clll}
\hline Policies & $\overline{\Delta g}(\%)$ & $\max \{\Delta g\}(\%)$ & $\Delta g<1 \%(\%)$ \\
\hline From lit. & & & \\
KB & 1.68 & 29.3 & 52.9 \\
FB & 2.36 & 32.4 & 36.2 \\
BSE & 4.02 & 67.4 & 37.4 \\
New & & & 58.6 \\
BSR & 1.58 & 26.5 & 35.2 \\
KBR & 3.13 & 35.2 & \\
\hline
\end{tabular}

by $x_{1}$ and $R$ (the inventory position of remanufacturable products) by $x_{2}$.

In the literature, three heuristic policies meet the above conditions:

The Kanban (KB) policy: $\left(\left[x_{1}+x_{2}^{+}\right]_{a},\left[x_{2}\right]_{r},\left[x_{2}\right]_{m}\right)$ which has been investigated by Korugan and Gupta (2000), see Fig. 13a.

The fixed buffer $(F B)$ policy: $\left(\left[x_{1}\right]_{a},\left[x_{2}\right]_{r},\left[x_{2}\right]_{m}\right)$ which has been investigated by van der Laan and Salomon (1997), see Fig. 13b.

The base stock echelon (BSE) policy: $\left(\left[x_{1}+x_{2}\right]_{a},\left[x_{2}\right]_{r},\left[x_{1}+x_{2}\right]_{m}\right)$ which has been investigated by Simpson (1978), see Fig. 13c.

In Sections 5.2 and 5.4 we observed that $\mu_{r}$ is a key parameter when choosing an efficient heuristic control. When $\mu_{r}$ is small, we observed that the preferred control rules are $\left[x_{1}+x_{2}^{+}\right]_{a},\left[x_{2}\right]_{r}$, and $\left[x_{2}\right]_{m}$. The policy with such control rules is the KB policy. In the same way, when $\mu_{r}$ is large, the preferred control rules are $\left[x_{1}+x_{2}\right]_{a},\left[x_{2}\right]_{r}$, and $\left[x_{1}+x_{2}\right]_{m}$. The policy with such control rules is the BSE policy.

For intermediate values of $\mu_{r}$, we look at two new combinations:

The base stock return (BSR) policy: $\left(\left[x_{1}+x_{2}\right]_{a},\left[x_{2}\right]_{r},\left[x_{2}\right]_{m}\right)$, see Fig. 14a.

The Kanban return $(K B R)$ policy: $\left(\left[x_{1}+x_{2}^{+}\right]_{a},\left[x_{2}\right]_{r},\left[x_{1}+x_{2}\right]_{m}\right)$, see Fig. 14b.

Table 4 summarizes our numerical results for these five heuristic policies.

We cannot claim that one heuristic policy outperforms all other heuristic policies. It is consistent with our analysis on each control rule. All control rules can have excellent results in some situations and poor results in other situations. Among the 6160 instances, one instance has a percentage cost increase of $8.5 \%$ for the five heuristic policies. Hence the optimal control can make a significant difference.

\section{Conclusion}

In this paper we have studied a hybrid manufacturing/remanufacturing system with limited capacity and stochastic demand, returns and processing times. We have partially characterized the structure of the optimal policy. Based on this characterization, we have selected several heuristic control rules for manufacturing, remanufacturing and returns acceptance. An extensive numerical study has lead to the following insights. On one hand, controlling remanufacturing with a fixed buffer control rule (using only local data) gives excellent results. On the other hand, the optimal manufacturing control and the optimal returns acceptance control can lead to large cost reductions in comparison with classic heuristic control rules that are used in the literature. Instance based heuristics can also lead to large cost increases.

We see several avenues for further research. Assumptions like no setup time and cost and exponential processing times could be relaxed. It would be also interesting to add the possibility to dispose accepted returns. Our model also assumes that preemption is allowed. Without preemption, the problem becomes significantly more complex and requires to add state variables to keep track of the state of the manufacturing and remanufacturing servers (on or off), offering another research direction.

\section{Acknowledgments}

One of the authors, SDF, wants to thank the European Union for financial support via the Erasmus staff exchange program.

\section{Appendix A. Proof of Theorem 1}

Because $\mathcal{T}$ is a contracting mapping, the fixed point theorem ensures that $v_{n+1}=\mathcal{T} v_{n}$ converges to the optimal value function $v^{*}$, which is the unique solution of the optimality equation $v^{\star}=T v^{\star}$ (Puterman, 1994). Moreover, $\mathcal{T}$ is a convex combination of cost function (C) and event operators denoted $T_{A(1)}, T_{C J(1,2)}$, and $T_{A(2)}$ in Koole (1998) for respectively $T_{a}, T_{r}$, and $T_{m}$. Koole (1998) proves that these operators propagate the properties of supermodularity and superconvexity. If we take $v_{0}\left(x_{1}, x_{2}\right)=0 \forall\left(x_{1}, x_{2}\right)$, it is clear that $v_{0} \in V$ and then via induction $v^{\star} \in V$.

As $v^{*} \in V$, supermodularity and superconvexity ensure that the three switching curves are well defined. For instance, convexity in $x_{2}$ ensures that we can define the manufacturing threshold $S_{m}\left(x_{1}\right)=\min \left[x_{1} \mid v\left(x_{1}, x_{2}+1\right)-v\left(x_{1}, x_{2}\right)+c^{m}>0\right]$. The monotonicity results for the switching curves are also implied by the fact that 
$v^{*} \in V$. For instance, supermodularity ensures that $S_{m}\left(x_{1}+1\right) \leq$ $S_{m}\left(x_{1}\right)$.

\section{Appendix B. Proof of Theorem 2}

We want to prove by induction that $\Delta_{\mathbf{e}_{2}-\mathbf{e}_{1}} v^{\star}\left(x_{1}, x_{2}\right)=$ $v^{\star}\left(x_{1}-1, x_{2}+1\right)-v^{\star}\left(x_{1}, x_{2}\right) \leq 0$. Let $v$ be the value function such that $\Delta_{\mathbf{e}_{2}-\mathbf{e}_{1}} v \leq 0$. We want to prove that $\Delta_{\mathbf{e}_{2}-\mathbf{e}_{1}} \mathcal{T} v \leq 0$.

Koole (2006) proves that operators $T_{a}, T_{r}$, and $T_{m}$ propagate the property $\Delta_{\mathbf{e}_{2}-\mathbf{e}_{1}} v \leq 0$. In addition, we have

$$
\begin{aligned}
\Delta_{\mathbf{e}_{2}-\mathbf{e}_{1}} c\left(x_{1}, x_{2}\right) & =c\left(x_{1}-1, x_{2}+1\right)-c\left(x_{1}, x_{2}\right) \\
& = \begin{cases}h_{1}-h_{2} \leq 0 & \text { if } x_{2} \geq 0, \\
-h_{1}-b \leq 0 & \text { else. }\end{cases}
\end{aligned}
$$

So we can conclude with (2) that $\Delta_{\mathbf{e}_{2}-\mathbf{e}_{1}} \mathcal{T} v \leq 0$ and by induction that $\Delta_{\mathbf{e}_{2}-\mathbf{e}_{1}} v^{\star} \leq 0$.

\section{Appendix C. System simplification}

The set of parameters is

$\left\{\delta, \mu_{r}, \mu_{m}, \lambda, h_{1}, h_{2}, b, c^{a}, c^{b}, c^{m}, c^{r}, \alpha\right\}$.

We want to simplify our model by reducing the number of parameters for the average cost criteria.

Let $f_{m}(\pi), f_{r}(\pi), f_{a}(\pi)$ and $f_{d}(\pi)$ be respectively the average flow of product from manufacturing, from remanufacturing, of accepted returns and of rejected returns. The average cost $C(\pi)=f_{m}(\pi) c^{m}+f_{d}(\pi) c^{b}+f_{a}(\pi) c^{a}+f_{r}(\pi) c^{r}+H(\pi)$, with $H(\pi)$ the average cost of storage and backlogs. We know that $f_{r}(\pi)=f_{a}(\pi)=\delta-f_{d}(\pi)$ and $f_{m}(\pi)=\lambda-\delta+f_{d}(\pi)$ so $C(\pi)=f_{a}(\pi)$ $\left(c^{a}-c^{b}+c^{r}-c^{m}\right)+\delta c^{b}+\lambda c^{m}+H(\pi)$.

We can set $c^{b}=c^{r}=c^{m}=0$ and create a relative acceptance cost $c=c^{a}-c^{b}+c^{r}-c^{m}$. Note that $c$ can be negative. The actual system has the same average cost optimal policy and its average cost is the same with a constant offset $\delta c^{b}+\lambda c^{m}$. This simplification does not hold for the discounted cost problem.

Moreover, we can set $\lambda=1$ and $h_{1}=1$ without loss of generality since it is equivalent to set a time and monetary units.

\section{Appendix D. Computational procedure}

To compute the optimal policy and the optimal cost, we truncate the state space in three directions. Let $M_{1}, M_{2}^{+}$and $M_{2}^{-}$ denote three integers with $0 \leq x_{1} \leq M_{1}$ and $M_{2}^{-} \leq x_{2} \leq M_{2}^{+}$. We apply a value iteration algorithm (Puterman, 1994) to this truncated state space and we increase the state space until the discounted/average cost is no more sensitive to the truncation level with 5 digits accuracy.
The approach to compute the cost of a heuristic policy is similar. We only need to change the operators. For instance, the operator of returns acceptance with heuristic control $\left[x_{1}+x_{2}\right]_{a}$ becomes

$\tilde{T}_{a} v\left(x_{1}, x_{2}\right)= \begin{cases}v\left(x_{1}+1, x_{2}\right) & \text { if } x_{1}+x_{2}<Z_{a} \\ v\left(x_{1}, x_{2}\right) & \text { else }\end{cases}$

When optimizing the policy parameters, we make the assumption that the expected cost is unimodal with respect to policy parameters. The assumption of unimodality has been checked on several instances.

\section{References}

Aras, N., Verter, V., Boyaci, T., 2006. Coordination and priority decisions in hybrid manufacturing/remanufacturing systems. Prod. Oper. Manag. 15 (4), 528-543.

DeCroix, G., 2006. Optimal policy for a multiechelon inventory system with remanufacturing. Oper. Res. 54 (May), 532-543.

Fleischmann, M., Kuik, R., Dekker, R., 2002. Controlling inventories with stochastic item returns: a basic model. Eur. J. Oper. Res. 138 (April (1)), 63-75.

Ilgin, M., Gupta, S., 2010. Environmentally conscious manufacturing and product recovery (ECMPRO): a review of the state of the art. J. Environ. Manag. 91 (January (3)), 563-591.

Inderfurth, K., 1997. Simple optimal replenishment and disposal policies for a product recovery system with leadtimes. OR Spektrum 19 (April (2)), 111-122.

Kiesmüller, G.P., 2003. A new approach for controlling a hybrid stochastic manufacturing/remanufacturing system with inventories and different leadtimes. Eur. J. Oper. Res. 147 (May (1)), 62-71.

Koole, G., 1998. Structural results for the control of queueing systems using eventbased dynamic programming. Queueing Syst. 30 (December (3)), 323-339.

Koole, G., 2006. Monotonicity in Markov reward and decision chains: theory and applications. Found. Trends Stoch. Syst. 1 (January), 1-76.

Korugan, A., Gupta, S., 2000. A Kanban control mechanism for a multi-echelon inventory system with returns. In: 2000 Annual Meeting of the Northeast Decision Sciences Institute, February, pp. 105-107.

Li, Y., Zhang, J., Chen, J., Cai, X., 2010. Optimal solution structure for multi-period production planning with returned products remanufacturing. Asia-Pac. J. Oper Res. 27 (October (5)), 629-648.

Liberopoulos, G., Dallery, Y., 2003. Comparative modelling of multi-stage production-inventory control policies with lot sizing. Int. J. Prod. Res. 41 (April (6)), 1273-1298.

Puterman, M.L., 1994. Markov Decision Processes, Discrete Stochastic, Dynamic Programming. John Wiley \& Sons, Inc..

Rubio, S., Chamorro, A., Miranda, F.J., 2008. Characteristics of the research on reverse logistics (1995-2005). Int. J. Prod. Res, 46 (February), 1099-1120.

Simpson, V., 1978. Optimum solution structure for a repairable inventory problem. Oper. Res. 26 (March (2)), 270-281.

Teunter, R.H., Vlachos, D., 2002. On the necessity of a disposal option for returned items that can be remanufactured. Int. J. Prod. Econ. 75 (February), 257-266.

van der Laan, E., Dekker, R., Salomon, M., 1996a. Product remanufacturing and disposal: a numerical comparison of alternative control strategies. Int. J. Prod. Econ. 45 (August (1-3)), 489-498.

van der Laan, E., Dekker, R., Salomon, M., Ridder, A., 1996b. An (s, Q) inventory model with remanufacturing and disposal. Int. J. Prod. Econ. 46-47 (December) 339-350.

van der Laan, E., Salomon, M., 1997. Production planning and inventory control with remanufacturing and disposal. Eur. J. Oper. Res. 102 (October (2)), 264-278.

van der Laan, E., Teunter, R., 2006. Simple heuristics for push and pull remanufacturing policies. Eur. J. Oper. Res. 175 (December (2)), 1084-1102. 